\title{
Arbor
}

\section{Le futur de l'Erope: un débat ouvert}

\section{Vlad Constantinesco}

Arbor CLXXII, 678 (Junio 2002), 227-238 pp.

Le futur de l'Europe n'a-t-il pas finalement été toujours ouvert, en débat et controversé? L'ouverture, la discussion et le pluralisme ne sontils pas, depuis toujours, les caractéristiques qui ont marqué l'histoire et la culture européennes? On ne doit donc pas s'étonner, aujourd'hui, de rencontrer ces traits, au moment où se dessine le futur de l'Union européenne, au moment où les travaux de la Convention débutent, avant de trouver peut-être leur consécration grâce à la Conférence intergouvernementale prévue en 2004.

Cependant, la question de l'avenir de l'Europe ne s'est jamais par le passé posée avec le relief particulier qu'elle prend aujourd'hui, et ce pour plusieurs raisons:

- On peut d'abord penser que le moment actuel n'est autre que celui qui a été depuis longtemps préparé par le chemin de l'intégration fonctionnelle pris à partir de la Déclaration Schuman du 9 mai 1950: cette déclaration traçait un programme qui devait mener, par la mise en commun d'intérêts nationaux à une fédération européenne. Le pari de faire naître une construction politique à partir d'une détermination économique, prenant en compte des tâches de plus en plus nombreuses exercées par les Etats membres (y compris dans des domaines régaliens) est peut-être justement en train de se réaliser maintenant. Ce retour (programmé?) du politique se marque précisément par le fait que les gouvernements des Etats membres ont accepté de modifier (jusqu'à, où?) la procédure de révision, en décidant, au Conseil européen de Laeken, de faire précéder la Conférence intergouvernementale par une Convention, selon une formule qui a permis la 


\section{Vlad Constantinesco}

rédaction de la Charte des droits fondamentaux de l'Union européenne. L'orientation vers un partage du pouvoir constituant (dont les proportions restent à déterminer), voulue par les gouvernements mêmes qui en détenaient jusque là le monopole, est un fait inédit. Il restera, bien sûr, à vérifier comment s'opérera l'articulation entre la Convention et la CIG: notamment, quelle autorité aura le texte de la Convention à l'égard de la CIG? A cet égard, le fait que la Convention se compose de délégués des gouvernements nationaux (voire de membres de ces gouvernements) et donc de personnes qui siégeront à la CIG fera que la CIG entrera dans la Convention. Et ceci peut être une garantie que si un accord existe au sein de la Convention, il aura des chances d'être repris par la CIG.

- L'ordre du jour de cette Convention a été élargi à Laeken, même si les points les plus importants et les plus difficiles demeurent ceux qui avaient été arrêtés par la déclaration $n^{\circ} 23$ annexée au traité de Nice. La discussion va en effet concerner tous les aspects délicats du stade acuel de l'intégration, même les plus controversés, tous ceux, finalement, sur lesquels il avait été impossible de s'entendre à Nice: la répartition des compétences, la simplification des traités, l'insertion des parlements nationaux dans l'architecture européenne et le statut de la Charte des droits fondamentaux de l'Union européenne. La nature et l'importance constitutionnelles de chacune de ces questions suffisent à montrer la singularité de ce moment qui devient, par l'objet même du débat un momentum constitutionnel que l'Europe n'a pas connu depuis 50 ans, depuis les travaux de l'Assemblée ad hoc, en 1952.

- Mais, autre singularité, le traité de Nice, dont on applique à l'avance la déclaration $n^{\circ} 23$, n'a pas été ratifié par tous les Etats membres, à la suite de la réponse négative de l'éléctorat irlandais au référendum en autorisant la ratification. Il n'est pas dit, par conséquent, que le traité de Nice entre un jour en vigueur, même si l'on peut espérer que les élécteurs irlandais reviendront sur leur première décision et surtout qu'ils se rendront plus nombreux aux urnes à l'occasion du second référendum annoncé pour le mois d'octobre 2002 . Si le traité de Nice n'obtenait pas la ratification de tous les Etats membres, alors la Convention et la Conférence intergouvernementale auraient à ajouter à leur ordre du jour les questions que Nice a entendu résoudre, à savoir les reliquats d'Amsterdam: re-pondération, composition de la Commission, vote 
à la majorité qualifiée, sans compter les sujets qui se sont additionnées en cours de route, comme la réforme des coopérations renforcées et l'allègement de leurs conditions de mise en œuvre. Un tel programme de travail deviendrait écrasant....

- La quatrième raison qui fait que la question de l'avenir de l'Europe se pose aujourd'hui avec une acuité particulière tient à l'élargissement. Pour une série de raisons, que l'on renonce à énoncer parce qu'elles sont connues, ce 5e élargissement ne ressemblera pas aux précédents. L'ambition du traité d 'Amsterdam, puis de celui de Nice, avaient été de préparer l'Union à l'élargissement: les réformes à introduire devaient permettre de maîtriser l'entrée d'une douzaine de pays, car une fois l'élargissement réalisé, l'unanimité, si elle était conservée pour la révision, empêcherait vraisemblablement toute évolution significative des traités. On reconnaît, d'une certaine façon, l'ancien dilemme: approfondissement ou élargissement. Or, il semble de plus en plus que les deux opérations, la CIG précédée de la Convention et l'élargissement seront menées parallèlement au lieu de se succéder. Il n'est pas sûr que la précipitation que la Commission à indiquer comme date prévisible de l'élargissement l'année 2004, c'est à dire l'année où s'engageront les travaux de la CIG, soit de bon augure pour la réussite de celle-ci, car le principe d'unanimité sera toujours en vigueur et l'augmentation du nombre d'Etats associés à la révision multipliera aussi les occasions de veto et le risque de solutions minimales.

Toutefois, si les conditions du débat sur l'avenir de l'Europe sont particulièrement propices à un réexamen de l'ensemble des éléments qui la configurent, il n'en demeure pas moins que les solutions d 'ensemble qui pourront guider les négociateurs ne sont pas vraiment nouvelles: c'est le contexte qui est nouveau, et toute la difficulté sera de vérifier si et comment ces solutions peuvent prétendre le traiter et encadrer ces nouvelles données et demandes.

Le leitmotiv commun se résume finalement à cette interrogation: quelle Europe voulons-nous construire ensemble? Pour y répondre, on se propose de décliner cette interrogation en trois questions successives, se résumant finalement à trois dilemmes:

- Première question, celle du contenu du projet européen: Europe puissance ou Europe marché? (I)

- Deuxième question, celle des structures: Confédération ou Fédération d'Etat nations? (II)

- Troisième question, celle des limites: l'Europe, jusqu'où? (III) 


\section{Vlad Constantinesco}

\section{Le contenu du projet européen: Europe puissance ou Europe marché?}

La question n'est pas nouvelle: elle avait été formulée il y a quelques années par M. Giscard d'Estaing. Elle consiste à se demander si l'ambition de l'Union européenne doit être de former, au sein de la mondialisation, un pôle de puissance économique et politique, ou si elle doit se contenter d'être le cadre d'organisation d'un grand marché, en limitant ses ambitions à l'encadrement de la libre circulation des marchandises, personnes, services et capitaux, accompagnée de quelques politiques communes revisitées..

Il faut bien entendu s'entendre sur ce que recouvre le mot puissance, qui n'est synonyme ni d'agressivité ni d'impérialisme. La puissance comprend évidemment une dimension de force et de richesse, projetées vers l'extérieur. Or ces deux dimensions existent bien dans l'Union européenne, mais à l'état latent, en quelque sorte:

- s'agissant de la force, deux Etats membres disposent de l'arme nucléaire et sont également membres permanents du Conseil de sécurité des Nations Unies, mais la Politique étrangère et de sécurité peine à rassembler les conceptions de ces Etats et celles des Etats, plus nombreux, qui ont affiché un statut de neutralité, sauf à considérer qu'un plus petit commun dénominateur entre les positions des Etats membres pourrait tenir lieu de politique extérieure commune. Sans doute la matière est-elle évolutive et certains signaux sont-ils intéressants: Saint-Malo, la décision prise à Nice (mais Nice n'est pas ratifié...) de constituer une force d'action rapide capable d'intervenir dans la zone de sécurité de l'Union, la mission de l'Union européenne en Macédoine. Mais inversement, on pourrait aussi multiplier les signes d'insuffisance: la réglementation contournée concernant le nouvel article $133 \mathrm{~T} \mathrm{CE}$ (politique commerciale commune) donnée à Nice ne facilitera pas l'interdépendance -pourtant nécessaire- entre les aspects économiques et les aspects politiques des relations extérieures de l'Union, le faible rôle joué par l'Union dans la crise israélo-palestinienne, en dépit des aides financières massives que l'Union apporte à la région, sa quasi absence de la crise tchétchène... On mesure ainsi le chemin qui reste à parcourir pour aboutir au plein exercice de cette dimension de la puissance.

- quant à la richesse, au développement économique de l'Union européenne, ils restent évidemment tributaires du dynamisme des Etats Unis, mais la création de la zone Euro a ici fondamentale- 
ment changé la donne. La monnaie commune est maintenant là et les européens, qu'ils soient opérateurs économiques ou particuliers peuvent maintenant en mesurer toute l'utilité et tout l'intérêt. Par le fait que l'Euro est choisi peu à peu comme monnaie de réserve, l'Union (en tous cas la zone Euro, apellée à s'élargir) sera en mesure de jouer un rôle déterminant face au dollar et au yen. Bien entendu, l'Euro souffre de l'absence d'un véritable pouvoir politique européen, et de la répartition des rôles arrêtée à Maastricht, à savoir une centralisation de la politique monétaire mais une simple coordination des politiques économiques des Etats membres. Le gouvernement économique de l'Union est encore dans les limbes, comme son gouvernement politique...Les conditions politiques et institutionnelles de la puissance économique comme de la puissance politique font défaut.

De là la tentation de considérer qu' à défaut de volonté de se doter des instruments de la puissance, l'Union peut plus facilement ne demeurer qu'un grand marché, la monnaie unique en étant le couronnement et l'aboutissement. Une telle conception peut alors se contenter d'un appareil institutionnel faible, et ne requiert pas de saut qualitatif constitutionnel. Bien au contraire, les piliers de coopération (PESC et JAI, qui concernent des prérogatives régaliennes des Etats) pourraient se transformer en un réseau d'accords seulement souscrits par les Etats intéressés, mais non applicables d'office à tous les Etats membres (une sorte de coopération renforcée a minima). Les contraintes juridiques pourraient être limitées aux règles d'organisation et de fonctionnement du marché intérieur, dont certaines politiques communes devraient être révisées, notamment sur le plan commercial externe, voire abandonnées telles qu'elles existent aujourdhui (politique agricole commune, fonds structurels). Elle s'accomoderait aussi probablement d'un nombre élevé d'Etats membres, plus exactement un nombre élevé d'Etats membres ne mettrait pas en cause la réalisation d'une Europe, simple espace d'échanges. La rançon d'une telle option, c'est évidemment la marginalisation de l'Union sur la scène internationale, et la réduction inévitable de son rôle d'amortisseur de la mondialisation. Une telle option ne peut pas tenir lieu d' ambition...

De la réponse à cete première question dépendent aussi les réponses aux autres questions. Sur ce point, un consensus semble déjà exister dans l'opinion publique: seuls ceux qui n'ont pas compris que le développement d'une Europe puissance est la seule garantie du maintien des Etats en tant que tels n'y sont pas favorables.

Naturellement, cette Europe puissance, fondée sur la réconciliation de la famille européenne, sur une culture de la paix et de la démocratie, 


\section{Vlad Constantinesco}

n'a pas à avoir d'ambition hégémonique: sa vocation est précisément de témoigner de ses valeurs, celles inscrites à l'art. $6 \S 1$ du traité sur l'Union: liberté, démocratie, respect des droits fondamentaux et de l'Etat de droit, valeurs développées et précisées par la Charte des droits fondamentaux de l'Union européenne.

\section{Les structures de l'Europe: Confédération ou Fédération d'Etat nations?}

On prétend souvent que les Communautés européennes se situeraient «quelque part» entre la Confédération d'Etats et la Fédération: elles se trouveraient donc dans une position intermédiaire et évolutive entre les deux formes examinées. Se situant au-delà d'une Confédération à certains égards, elles n'auraient cependant pas (encore?) atteint le stade -ultime?- de la Fédération. Considérées du point de vue institutionnel et décisionnel, les Communautés européennes relèvent en effet simultanément des deux catégories: Fédération et Confédération constituent bien, ensemble et de manière complémentaire, les deux modèles explicatifs susceptibles d'en rendre compte, chacun pour partie.

Ainsi, l'Union et les Communautés européennes ne relèvent pas clairement de l'une ou de l'autre des formes politiques classiques que sont l'Etat fédéral ou la Confédération d'Etats: à certains égards, elles se trouvent dans une situation ambigue et hybride de mixtum compositum, empruntant à chacune de ces formes certains éléments qui coexistent selon un dosage complexe et dont la part respective varie selon les secteurs retenus. Rien d'étonnant alors à ce que la perception de l'édifice par l'opinion publique soit brouillée et confuse.

Dès lors doit-on rester dans cet entre-deux ou faut-il clarifier et opter soit pour une simple Confédération d 'Etats, soit pour une Fédération? Le dilemme est ici celui qui oppose les » souverainistes » et les » fédéralistes «. La question qui les divise, et qui divise aussi la plupart des partis politiques, est celle de la souveraineté. Car la Confédération repose sur la souveraineté des Etats membres et lui est subordonnée, tandis qu'au contraire, la Fédération (sous la forme de l'Etat fédéral) déplace la souveraineté des Etats membres vers les institutions centrales, ceux-ci cessant d'être souverains.

Aussi la formule de Jacques DELORS, la Fédération d'Etats nations, paraît-elle attractive pour dépasser ce dilemme et pour proposer une architecture juridique et politique à l'Union européenne. Il faut se demander si la formule possède un sens, ou si elle n'est après tout qu'habile rhé- 
torique, simplement destinée à faciliter un changement qui, sous des apparences de changement majeur, n'en serait finalement pas un. Que veut-on dire, exactement, en parlant d'une Fédération des Etats-nations?

L'Etat-nation était ainsi défini par Denis de ROUGEMONT: «L'Etatnation, né de la guerre et progressant par elle, comme elle par lui, conduit nécessairement à de nouveaux conflits qu'il prépare sous le nom de défense de la Paix. Et ce seront les deux guerres mondiales.» L'Etat-nation a partie liée avec le nationalisme et l'on sait, comme le disait F. MITTERRAND, que: «le nationalisme, c'est la guerre!» Si tel est le cas c'est parce que l'Etat-nation revendique et exerce pleinement, sans contrôle ni limites, la souveraineté. Autrement dit, le terme d'Etat-nation renvoie à celui d'Etat souverain. Or, selon la doctrine classique de l'Etat fédéral, les États membres de l'Etat fédéral, une fois le Pacte fédéral conclu, cessent d'être souverains pour s'assujettir volontairement à un ordre auquel il participent, mais dont ils sont les sujets. Indiquer que l'Europe devrait devenir une Fédération des Etats-nations, c'est donc admettre la coexistence de deux niveaux de souveraineté, ou considérer celle-ci comme divisible, ce qui contredit le postulat de l'unité et de l'indivisibilité de la souveraineté. L'expression porterait alors en elle même la contradiction: ce ne serait qu'un oxymore politique.

Toutefois, cette contradiction serait relativisée et l'expression «Fédération des Etats-nations» recevrait une certaine cohérence si on considérait, dans la lignée de Georges SCELLE, la souveraineté comme un faisceau de compétences et comme un titre juridique permettant d'en organiser l'exercice en commun par des institutions communes établies par les Etats. Dès lors, l'expression s'éclaire, car elle suppose que les compétences de la Fédération soient clairement énoncées et délimitées par le Pacte fédératif (qui, pour J.DELORS, doit demeurer un traité international et pas nécessairement prendre la forme d'une constitution), le même pacte réservant aux Etats-nations des compétences exclusives recouvrant l'ensemble des questions liées à la cohérence d'une nation et d'une société: l'emploi, le social, la santé, l'éducation et la culture.

Le succès de la formule ne repose pas uniquement sur l' équivoque: il est du au fait qu'elle exprime assez justement non seulement le projet européen, qui doit certes encore être matérialisé et concrétisé, peut-être sous la forme d'une constitution, mais aussi -et surtout- parce qu'elle est en phase avec la réalité communautaire d'aujourd'hui, telle que des années de pratique institutionnelle et étatique, de débats aussi, l'ont façonnée. Il est vrai que la réalité communautaire combine les éléments fédéraux et confédéraux dans une proportion difficile à établir, car elle varie d'intensité sans doute selon les secteurs et les critères retenus. 
Le point noir de la formule tient, pour beaucoup, à ce qu'elle semble consacrer l'immuabilité des 'Etats nations', tels que les histoires nationales les ont forgés, avec les inflexions que chaque Etat membre a pu connaître dans sa recherche d'une relation particulière entre l'Etat et la nation. Mettre l'accent sur les 'Etats nations', comme composantes de la Fédération, c'est aussi écarter toute perspective d'une Fédération de régions, c'est consolider l'Etat nation' comme véhicule d'une conception unitaire de la stato-nation, c'est aussi récuser des modes d'organisation collective qui, comme en Espagne ou en Belgique et, dans une moindre mesure, en Italie, tiennent compte d'un pluralisme national ou nationalitaire.

Il a pu y avoir (historiquement et doctrinalement) une certaine antinomie entre le fédéralisme et l'Etat unitaire, voire l'Etat tout court. L'Etat a pu voir, à maintes reprises depuis un demi-siècle de construction européenne, sa mort annoncée: pris en tenaille entre une construction européenne qui le dépouille de ses prérogatives régaliennes et l'essor d'un régionalisme qui lui soustrait de plus en plus de compétences de gestion, l'Etat n'est-il pas appelé à se vider de sa substance, par le «haut» comme par le «bas»?

La formule Fédération d'Etats nations se veut pragmatique et possibiliste: il ne peut s'agir de poursuivre et d'approfondir la construction de l'Union et des Communautés européennes contre les Etats, ni avec d'autre partenaires publics qu'eux. Sans doute, les régions, spécialement celles à statut constitutionnel et à compétence législative, revendiquentelles une plus grande présence (et une présence plus effective) dans les institutions et les mécanismes décisionnels de l'Union. Leur crainte est ici symétrique de celle évoquée à l'instant: ils redoutent que le développement des domaines d'action de l'Union et des Communautés n'aboutisse à mettre en cause les compétences que les constitutions des Etats leurs reconnaissent.

Mais s'il convient d'être attentif aux demandes du Comité des régions ou de la conférence des régions à statut constitutionnel, voire de reconnaître la justesse de certaines (comme par exemple le droit, pour les régions, de saisir la Cour de justice), il semble difficile, et c'est à notre avis le sens de la formule d'une «Fédération d'Etats nations", d'imaginer que les Etats membres acceptent de renoncer demain au principe de leur monopole de la représentation des nations dont ils tirent leur légitimité. Pour le dire encore plus clairement: est-il acceptable et imaginable que les Etats membres redistribuent toutes leurs compétences entre les niveaux plus proches des citoyens et le niveau de l'Union européenne, et qu'ils s'effacent devant les régions et l'Europe? 


\section{Le futur de l'Europe: un débat ouvert}

Plus exactement, si des aménagements doivent être trouvés quant à l'association des régions aux mécanismes décisionnels communautaires et en particulier au fonctionnement du Conseil de l'Union européenne, il s'agit avant tout de solutions que le droit national est habilité à trouver et à donner, plus que le droit communautaire. (la composition des délégations nationales au Conseil peut comprendre d'autres personnes que les ministres des gouvernements nationaux, comme la rédaction de l'art. $204 \mathrm{~T} \mathrm{CE}$ le permet et comme le montrent les pratiques suivies par l'Allemagne et la Belgique.

L'Union de demain, dont les traités constitutifs auront été rationalisés et restructurés, qui bénéficiera peut-être de la personnalité juridique, continuera de reposer sur d'anciennes nations qui n'entendent ni se fondre ni éclater en communautés multiples et diverses. Elle continuera aussi d'être cet ensemble plurinational, où certaines compétences étatiques seront exercées en commun conformément au principe de subsidiarité, selon des procédures simplifiées et plus transparentes impliquant les diverses institutions, chacune exprimant un des grands intérêts associés. Mais les gouvernements des Etats membres devront prendre garde à modifier la procédure de révision des traités, à augmenter l'agilité décisionnelle d'une Union élargie et alourdie, en diffusant le vote à la majorité qualifiée, à préciser ce qu'est le modèle social européen, à faire en sorte que le citoyen européen trouve des raisons positives d'apporter un soutien actif à l'une des entreprises les plus spectaculaires du siècle qui s'achève. Proudhon avait prédit que le XXe siècle serait celui de la Fédération, sinon l'humanité recommencerait un purgatoire de mille ans: que nous apportera le XXIe siècle?

L'Union européenne sera-t-elle finalement une Fédération? une Confédération? La constitution américaine ne comporte pas le mot fédéral, et elle fonctionne depuis plus de deux siècles; la constitution suisse dont la dénomination officielle est la constitution fédérale de la Confédération hélvétique, de 1874, vient de se voir renouvelée par une révision générale en 1999: si l'Union doit inventer son propre modèle, qu'elle ne se laisse pas paralyser par des étiquettes!

\section{Les limites de l'Europe:}

Cette dernière question est loin d'être la plus simple, même si on peut penser qu'avec l'élargissement programmé l'Union a déjà commencé à y donner une réponse. Tous les Etats candidats qui ont ouvert des négociations avec l'Union peuvent être considérés comme des Etats eu- 


\section{Vlad Constantinesco}

ropéens, au sens de la géographie, de l'histoire et de la culture. En revanche, la Turquie, dont le principe de l'adhésion a été admis au moment du premier accord d'association, mais pour laquelle les négociations n'ont pas encore commencé pose un difficile problème. Derrière la Turquie se profilent d'autres candidatures: Ukraine, Biélorussie, Russie elle-même peut-être un jour...

La réponse à cette question est complexe et dépend de plusieurs facteurs:

- du point de vue juridique, le traité sur l'Union ne contient qu'une seule condition, assez lâche et assez imprécise: tout Etat européen peut faire acte d'adhésion. Comment interpréter cette condition? Si la réponse est purement géographique, on considérera que la Turquie n'a qu'une partie fort réduite de son territoire sur le continent européen, mais si la réponse est d'ordre géo-politique, on conviendra que la Turquie est, depuis les origines de ces organisations, membre de l'OTAN et du Conseil de l'Europe: de ce point de vue, elle est alors un Etat européen. En tant que tel, la Turquie est depuis son adhésion au Conseil de l'Europe, soumise au contrôle de la Cour européenne des droits de l'homme, c'est à dire à un mécanisme juridictionnel de surveillance et de contrôle des obligations conventionnelles qu'elle a acceptées. Ainsi peut se vérifier, en dehors de l'Union, que cet Etat candidat est bien à même de respecter les conditions d'ordre politique qui conditionnent l'adhésion à l'Union.

- du point de vue politique, on peut se référer à la Déclaration de Copenhague (1993) et à l'Agenda 2000 présenté par la Commission en 1997, documents qu'il faut compléter par les conditions établies par l'art. $6 \$ 1$ du traité sur l'Union. Mais d'autres conditions, comme l'existence d'une économie de marché, l'infrastructure administrative et juridique, l'acceptation de l'ensemble de l'acquis communautaire doivent aussi être remplies par les Etats candidats. C'est donc à l'Etat candidat que revient le soin de démontrer qu'il remplit toutes ces conditions. C'est en réalité l'Etat candidat qui fixe la date de son adhésion. A cet égard,

- la présence sur le territoire de l'Union, particulièrement en Allemagne, d'une forte communauté turque, qui génère des flux financiers importants, est également un élément à prendre en considération: sur le plan financier, la Turquie n'est-elle pas déjà le $16^{\mathrm{e}}$ Etat membre (invisible) de l'Union?

- la procédure d'admission repose sur le double consentement de tous les Etats et sur l'avis conforme du Parlement européen, 
donné à la majorité absolue de ses membres. Chaque Etat et la majorité du Parlement européen disposent ainsi d'un droit de veto. Un double verrou communautaire est ainsi en place.

Ces éléments rappelés, comment répondre à la question de l'admission de la Turquie et d'autres Etats, aux marges de l'Union actuelle, qui manifesteraient la même volonté? La réponse prend en réalité la forme d'un pari et peut s'appuyer sur des précédents qu'une organisation comme le Conseil de l'Europe a déjà pratiqué, avec des conclusions mitigées...

Le pari est le suivant: face à ce type de candidature l'organisation a le choix entre l'acceptation de l'adhésion et le sursis à statuer. L'acceptation se justifie par l'argumentation selon laquelle il vaut mieux que l'Etat candidat soit à l'intérieur de l'organisation, dont il aura accepté les règles, et dont il pourra ainsi subir le contrôle et éventuellement la pression, voire la contrainte. Le sursis à statuer permet à l'organisation d'exercer une pression sur l'Etat candidat jusqu'à ce qu'il ait donné de sérieux gages de sa volonté et de sa capacité à assumer les obligations qu'il entend contracter.

$\mathrm{Au}$.fond, la réponse dépend surtout d'une considérations de bon sens selon laquelle il y a une différence naturelle entre le membre de la famille et le voisin. Le membre de la famille partage un héritage commun, des valeurs et des comportements qui lui ont été transmis de génération en génération; le voisin, lui, est proche et il vaut mieux avoir avec lui de bonnes relations, mais celles-ci n'atteindront jamais toutefois l'intensité de celles qui tissent les liens familiaux.

L'Union aurait donc tout intérêt à construire un statut juridique destiné à toutes ses zones de proximité: Maghreb, Méditerranée orientale, Balkans... Ce statut devrait aller au delà des formules d'association éprouvées jusqu'à présent, mais demeurer en deça de l'adhésion. La formule des accords partiels, comme ceux qui lient la Suisse à l'Union, pourrait servir d'inspiration. Car beaucoup de ces Etats (et le raisonnement eût pu être le même pour bon nombre d'actuels candidats à l'adhésion, en 1990) désirent un rapprochement avec l'Union, mais ne sont pas pour autant prêts à souscrire à l'ensemble des engagements que suppose une adhésion, ou ne le souhaitent pas.

Mais une réflexion sur les limites de l'Europe, simplement esquissée ici, ne peut pas faire l'économie d'un constat et d'une question, qui resteront peut-être sans réponse, du moins immédiate...

Le constat est celui d'un enchevêtrement complexe de multiples organisations européennes, comprenant parfois des Etats non-européens (OTAN, UEO, OECD, Conseil de l'Europe, OSCE, etc...) qui donne non 


\section{Vlad Constantinesco}

seulement l'impression de chevauchements et de doubles emplois, mais qui brouille la perception qu'ont les citoyens de la construction européenne. Sans doute cette situation est l'héritage de notre histoire et chacune de ces organisations constitue une strate historique de la longue marche de notre continent vers son unité. Sans doute cette multiplicité de structures n'est-elle ni rationnelle ni efficace: mais c'est notre réalité plurielle telle que le temps l'a façonnée.

La question découle du constat: comment faire? que faire? pour améliorer l'inter opérativité de ces organisations, en sachant que la plus puissante, l'Union européenne, a une tendance naturelle à étendre ses activités, notamment par rapport à celles du Conseil de l'Europe, en matière de droits de l'homme? Comment introduire un minimum de cohérence dans ce paysage touffu et déroutant? L'élargissement de l'Union va donner aux Etats membres de l'Union la majorité au Conseil de l'Europe: une réflexion devrait d'ores et déjà être menée pour tirer toutes les implications de ce fait et pour réfléchir, en termes de complémentarité, au sort d'un Conseil de l'Europe susceptible d'être contrôlé indirectement par l'Union. Notamment, si la Charte des droits fondamentaux de l'Union européenne acquiert force obligatoire au sein de l'Union, ne faut-il pas reconsidérer la question de l'adhésion de l'Union (à condition qu'elle reçoive enfin une personnalité juridique expresse) et de la Communauté à la Convention européenne des droits de l'homme? Et peut-être même au Statut du Conseil de l'Europe? Ce serait un premier pas d'une démarche de rationalisation, qui doit être bien pensée avant d'être entreprise, et qui mettrait fin à une confusion, et à une rivalité qui n'ont pas lieu d'être.

$$
\text { * * * }
$$

Les échéances prochaines qui attendent l'Union: Convention, CIG, élargissement sont, chacune en elles-mêmes, décisives. Leur accumulation et leur imbrication présente un caractère inédit dans la mesure où cette interdépendance entre un processus constitutionnel et un processus d 'élargissement, tous deux étant des opérations hautement complexes, peut déboucher sur les risques d'un emballement, et sur une fuite en avant non maîtrisée. Mais le pire n'est jamais totalement sûr: ce momentum constitutionnel d'une Union européenne élargie est aussi un formidable défi à la volonté et à l'imagination des européens. Le moment est venu d'en user! 